\title{
A Comparison of Low Reynolds Number $k-\varepsilon$ Models
}

\author{
Nie Xin ${ }^{1, a}$, Li Lei $^{2, b}$ \\ ${ }^{1}$ College of Mechanical Engineering,Hangzhou Dianzi University , 310018, China \\ ${ }^{2}$ College of Mechanical Engineering,Hangzhou Dianzi University , 310018, China \\ axin_nie2000@163.com, ${ }^{b} 510038771 @ q q . c o m$
}

Keyword: Low Reynolds number $k-\varepsilon$ turbulence model,Standard $k-\varepsilon$ turbulence model,the near-wall shear flow.

\begin{abstract}
Low Reynolds number $k-\varepsilon$ turbulence model is gained by modifying the standard $k-\varepsilon$ turbulence model, it applies to the whole fluid region. This case was studied six low Reynolds number $k-\varepsilon$ turbulence model and standard $k-\varepsilon$ turbulence model in FLUENT14.0 for numerical calculations near-wall shear flow,through comparing the speed, thus it is concluded that low Reynolds number $k-\varepsilon$ turbulence model is more accurate than the standard $k-\varepsilon$ turbulence model.In the low Reynolds number $k-\varepsilon$ turbulence model, LS, YS model compared to several other model, calculated value and actual value deviation is bigger.
\end{abstract}

\section{Introduction}

For the near-wall boundary layer flow and jets,early turbulent is based on Prandtl mixing length model assumptions and Boussinesq eddy viscosity hypothesis.Subsequently, Kolmogorov and Rotta proposed turbulence model is based on partial differential equations, due to restrictions of the computer technology leads to the equation can not be solved. A lot of turbulence models have been developed based on these two assumptions, and they have been widely used in a variety of flow forecast.Researchers have proposed a wide variety of two-equation model, three models of which the most widely used is the $k-\varepsilon$ model, the $k-\omega$ model and the $k-\omega-S S T$ model.

The low Reynolds number $k-\varepsilon$ turbulence model is obtained by amending the standard $k-\varepsilon$ turbulence model , and fit most flow calculations.

Features of low Reynolds number turbulence is affecting of the molecular viscosity to flow relatively increased.it not only affects the transport mean flow, and directly and indirectly affect various turbulent processes.In view of the industrial process is that the most common low Reynolds number turbulence occurs in the near-wall region, and therefore focuses on the low Reynolds number turbulence following the near-wall region.

In the vast majority of high Reynolds number turbulence, need considered low Reynolds number turbulence thickness near the wall zone does not exceed $2 \%$ of the system cross-sectional dimension.In the near-wall region, production rate of the turbulent kinetic energy $k$ and turbulent kinetic energy dissipation rate $\varepsilon$ reaches a maximum, approximately it's on the local equilibrium;Average flow velocity and temperature of the second derivative is large, which means all traffic gradient is large;Parallel to the wall shear stress may be a constant ,and equal to the wall stress $\tau_{w}$.In addition to these facts, by simple analysis, but also provide some interesting features near-wall region of turbulence.

\section{Numerical Methods}

This example is the application of seven different turbulent models to simulate the near-wall shear flow.FLUENT14.0 is used as the RANS solver for numerical investigations.Flow field consists of rectangular cross-section and smooth groove wall,and a plate at the entrance split into two imports. The flat is from the wall $b=0.005 \mathrm{~m}$ (inlet $1: v=2 \mathrm{~m} / \mathrm{s}$,inlet $2: v_{\max }=10 \mathrm{~m} / \mathrm{s}$ ), Working medium is $\operatorname{air}\left(\rho=1.225 \mathrm{~kg} / \mathrm{m}^{3}, \mu=1.7894 \times 10^{-5} \mathrm{~kg} / \mathrm{m} \cdot \mathrm{s}\right)$. The channel length is $0.41 \mathrm{~m}$, width is $0.15 \mathrm{~m}$, height is $0.1 \mathrm{~m}$ (Sampling line $l_{1 \sim 8}=0.03 \mathrm{~m}$,position shown in Fig. 1 , in $\mathrm{mm}$ ). 
Detailed grid independence study was conducted in order to ensure accurate results.The grid “m0",initially, total number of cells was taken as 2302800.Then, refine the mesh to give several other grids.By calculating different grid, obtain different results, and comparison.Figure 2 shows the effect of the grid independence on the velocity variations. As can be seen, in a certain range, the velocity of the impact of the distribution grid is weak[10].This study focuses on the low Reynolds number turbulence stage, therefore, the results given by the intersection of data from the distance between the two beams ranging $5 \mathrm{~mm}$ to $250 \mathrm{~mm}$ eight observation line.From the first grid node at the wall $y^{+}=4 \sim 9\left(y^{+}=y u_{\tau} / v, u_{\tau}\right.$ is friction velocity), That $y^{+}$meet the criteria of low Reynolds number turbulence models.

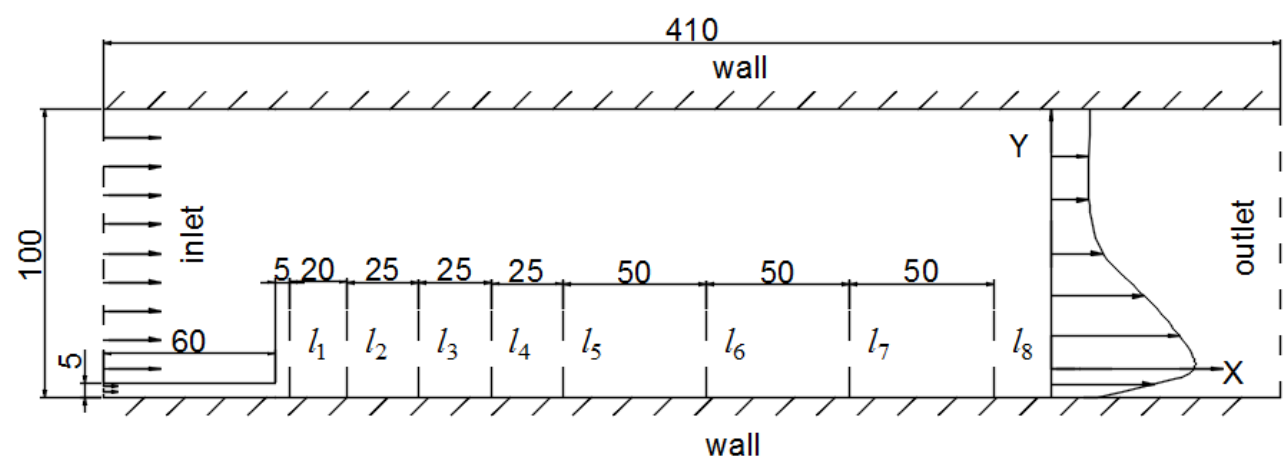

Fig. 1 The flow path geometry diagrams

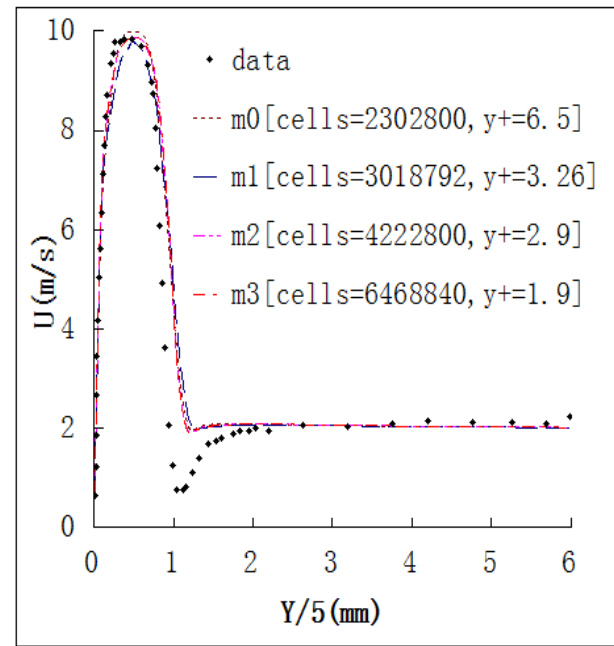

Fig. 2 Effect of grid refinement on self-similar velocity profile

The continuity and momentum transport equations as long as Reynolds stress closure equations are solved for the computational domain.

Continuity equation:

$\frac{\partial U_{i}}{\partial x_{i}}=0$

Momentum equation:

$\frac{D U_{i}}{D t}=-\frac{1}{\rho} \frac{\partial P}{\partial x_{i}}+\frac{\partial}{\partial x_{i}}\left(v \frac{\partial U_{i}}{\partial x_{j}}-\overline{u_{i} u_{j}}\right)$

Where linear eddy-viscosity model uses the relationship between stress and strain as follows:

$$
\overline{u_{i} u_{j}}=2 / 3 k \delta_{i j}-v_{t}\left(\frac{\partial U_{i}}{\partial x_{j}}+\frac{\partial U_{j}}{\partial x_{i}}\right)
$$

Where $v_{t}$, it is by solving a set of turbulent transport equations resulting eddy viscosity.

Low Reynolds number turbulence model solution based on the turbulent kinetic energy and its dissipation rate transport equation, as follows: 


$$
\begin{aligned}
& \frac{D k}{D t}=\frac{\partial}{\partial x_{j}}\left[\left(v+\frac{v_{t}}{\sigma_{k}}\right) \frac{\partial k}{\partial x_{j}}\right]+P_{k}-\tilde{\varepsilon}-D \\
& \frac{D \tilde{\varepsilon}}{D t}=\frac{\partial}{\partial x_{j}}\left[\left(v+\frac{v_{t}}{\sigma_{\varepsilon}}\right) \frac{\partial \tilde{\varepsilon}}{\partial x_{j}}\right]+C_{\varepsilon 1} f_{1} \frac{1}{T_{t}} P_{k}-C_{\varepsilon 2} f_{2} \frac{\tilde{\varepsilon}}{T_{t}}+E
\end{aligned}
$$

Where $f_{\mu}, f_{1}, f_{2}, \tilde{\varepsilon}, D$ and $E$ are vary with the given models, which $f_{\mu}, f_{1}$ and $f_{2}$ are the local turbulent model damping functions, $\tilde{\varepsilon}$ and $\varepsilon$ is related, $D$ and $E$ is an additional source term, $P_{k}$ is the turbulent kinetic energy produced items.Eddy viscosity in the $k-\varepsilon$ model is defined as $v_{t}$ $=C_{\mu} f_{\mu} k^{2} / \varepsilon \cdot C_{\mu}$ is a constant and $f_{\mu}$ is damping functions.In Equation (5) $T_{t}$ is the length of time of turbulence , $\tilde{\varepsilon}$ is isotropic dissipation rate [7].

Table 1 Low Reynolds number $k-\varepsilon$ models and their model constants

\begin{tabular}{cccccc}
\hline Model & $C_{\mu}$ & $C_{\varepsilon 1}$ & $C_{\varepsilon 2}$ & $\sigma_{k}$ & $\sigma_{\varepsilon}$ \\
\hline SKE & 0.09 & 1.45 & 1.92 & 1.0 & 1.3 \\
AB & 0.09 & 1.44 & 1.83 & 1.0 & 1.4 \\
LB & 0.09 & 1.44 & 1.92 & 1.0 & 1.3 \\
LS & 0.09 & 1.44 & 1.92 & 1.0 & 1.3 \\
YS & 0.09 & 1.44 & 1.92 & 1.0 & 1.3 \\
AKN & 0.09 & 1.5 & 1.90 & 1.4 & 1.4 \\
CHC & 0.09 & 1.44 & 1.92 & 1.0 & 1.3 \\
\hline
\end{tabular}

Table 2 The damping functions used in the Low Re. turbulence model

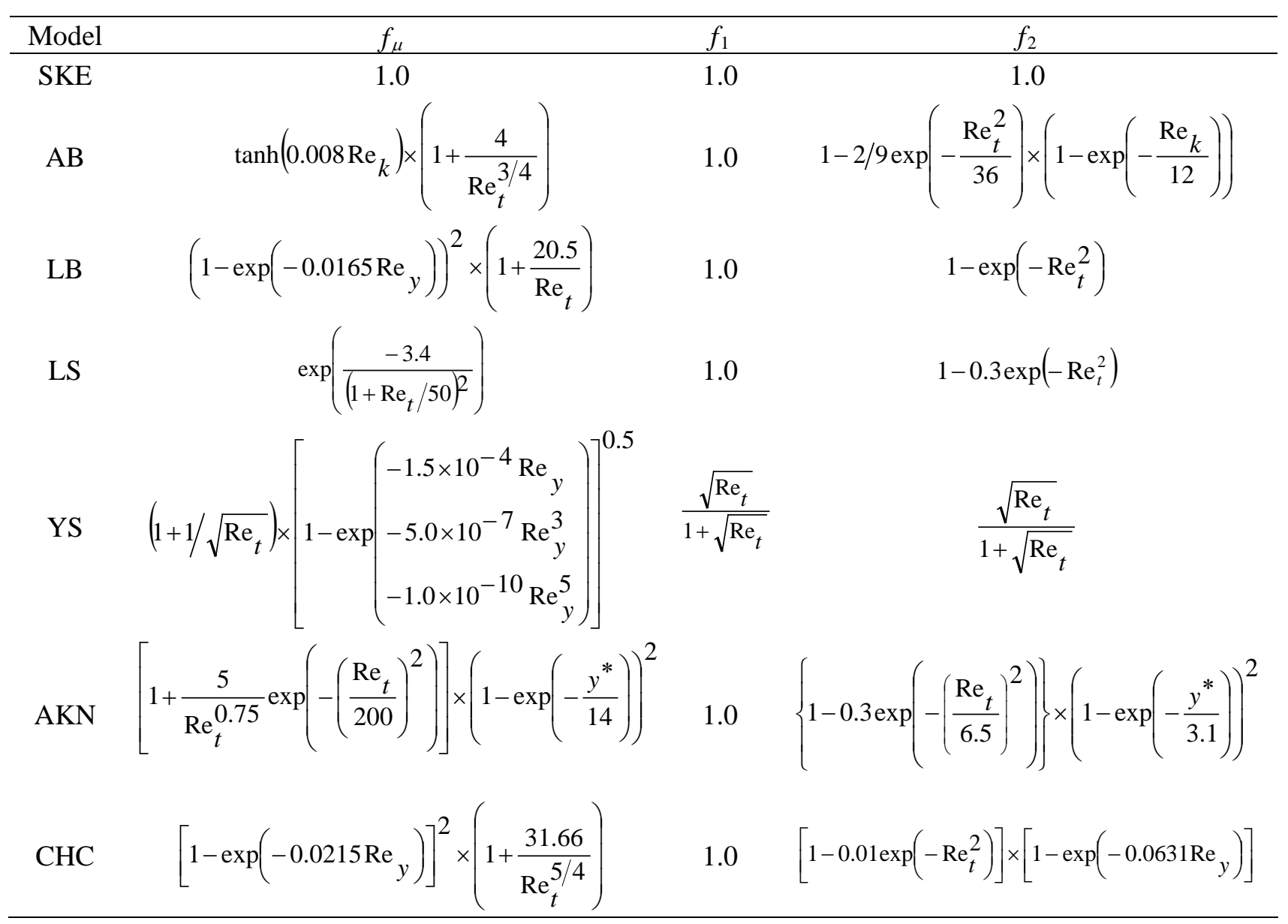


Seven turbulence models, namely those of Standard $k-\varepsilon$ ( SKE, Launder and Spalding (1974)) [8], AB ( Abid (1991)) [3], AKN ( Abe et al. (1994)) [6], CHC ( Chang et al. (1995)) [1], LB ( Lam and Bremhorst (1981)) [4], LS ( Launder and Sharma (1974)) [2], YS (Yang et al. (1993)) [5].

Each model coefficients, damping functions, and near-wall correction functions are listed in Table 1-3.

Table 3 Additional terms and wall boundary conditions for Low Re. Turbulence models

\begin{tabular}{cccc}
\hline Model & $D$ & $E$ & Wall B.C. \\
\hline SKE & 0 & 0 & Wall functions \\
AB & 0 & 0 & $\varepsilon_{w}=v\left(\frac{\partial^{2} k}{\partial y^{2}}\right)$ \\
LB & 0 & 0 & $\varepsilon_{w}=v\left(\frac{\partial^{2} k}{\partial y^{2}}\right)$ \\
LS & $2 v\left(\frac{\partial \sqrt{k}}{\partial y}\right)^{2}$ & $2 v v_{t}\left(\frac{\partial^{2} U}{\partial y^{2}}\right)^{2}$ & $\tilde{\varepsilon}_{w}=0$ \\
YS & 0 & $v v_{t}\left(\frac{\partial^{2} U}{\partial y^{2}}\right)^{2}$ & $\varepsilon_{w}=v\left(\frac{\partial^{2} k}{\partial y^{2}}\right)$ \\
AKN & 0 & 0 & $\varepsilon_{w}=v\left(\frac{\partial \sqrt{k}}{\partial y}\right)^{2}$ \\
CHC & 0 & 0 & $\varepsilon_{w}=v\left(\frac{\partial^{2} k}{\partial y^{2}}\right)$ \\
\hline
\end{tabular}

Note: $\operatorname{Re}_{t}=\frac{k^{2}}{v \varepsilon}, \operatorname{Re}_{y}=\frac{y k^{1 / 2}}{v}, y^{*}=\frac{y u_{\varepsilon}}{v}, u_{\varepsilon}=(v \varepsilon)^{0.25}, \widetilde{\varepsilon}=\varepsilon+D$.

\section{Analysis}

Figure 3 is a comparison of six low Reynolds number $k-\varepsilon$ turbulence model and the standard $k-\varepsilon$ turbulence model along the flow of the velocity calculated and experimental data[11].From the figure we can conclude that the SKE,YS model and experimental data slightly different, other models are in good agreement with the experimental data.At the $x / b=1,5,10,15$,in turbulence generation stage, seven kinds of $k-\varepsilon$ turbulence model results with the experimental data large deviations.At the $x / b=1,5$,in the seven kinds of $k-\varepsilon$ turbulence models speed reduced slower than the experimental values, while at $x / b=10,15$,in the seven kinds of $k-\varepsilon$ turbulence models speed reduced faster than the experimental values, indicating in the seven kinds of $k-\varepsilon$ turbulence models simulation of the junction of two jets are biased.In the vicinity of the wall, the results of six low Reynolds number $k-\varepsilon$ turbulence model is better than the SKE model results, mainly due to the low Reynolds number $k-\varepsilon$ turbulence model reduces the viscosity near the wall, and enhance the molecular viscosity, and lead to the results closer to the experimental value. In YS model maximum speed is higher than the calculated values of other models and the experimental values, you can see the role of the damping function, and due to the reduced rate of dissipation, high speed than the experimental values.

Overall, calculated value of the low Reynolds number $k-\varepsilon$ turbulence model near the wall than standard turbulence model is more accurate;in the low Reynolds number $k-\varepsilon$ turbulence models, SKE,LS and YS model compared with several other model,and the precision is low; Several models simulation and actual fluid meets the accuracy requirements, with engineering value 

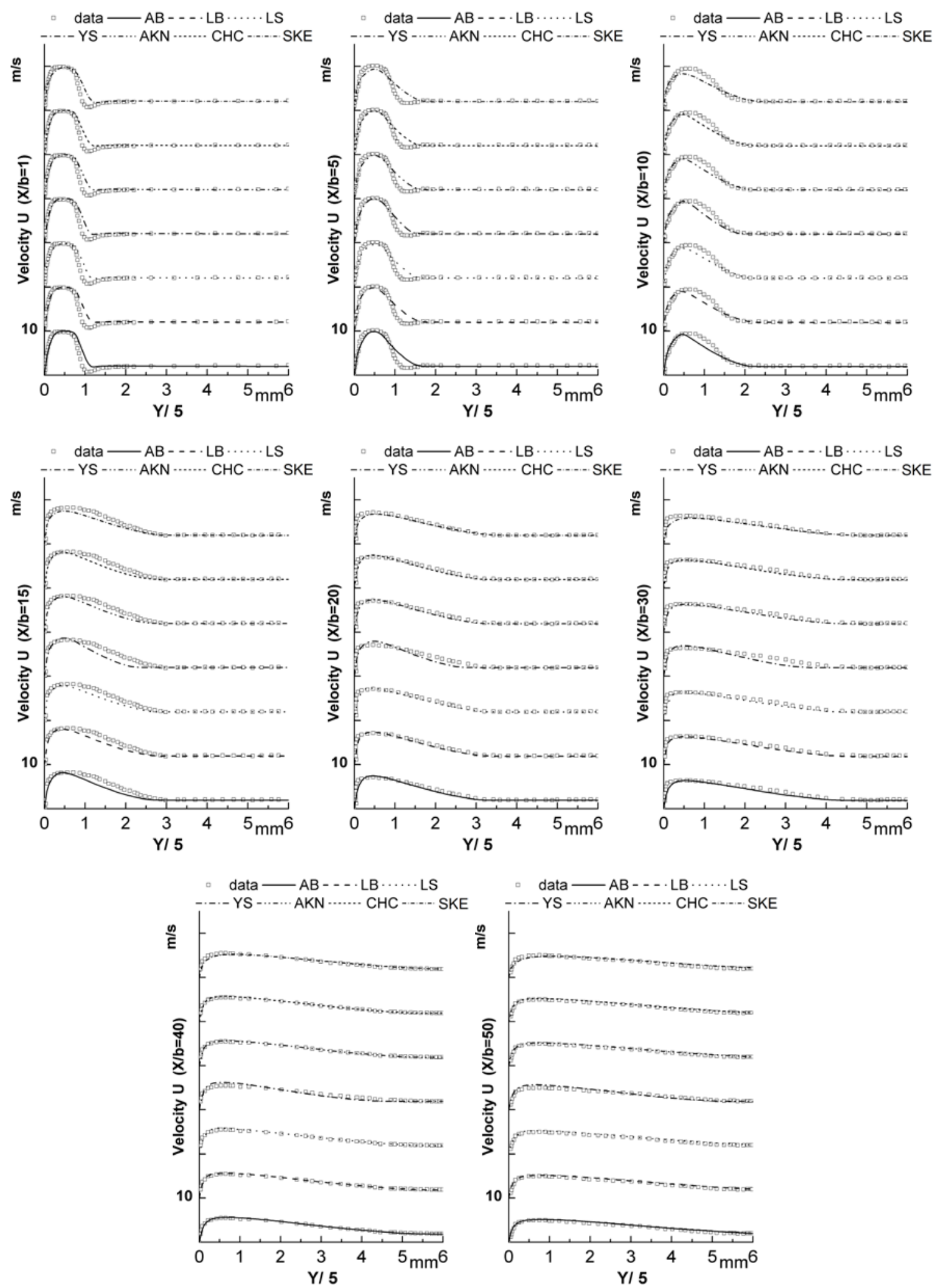

Fig. 3 Comparison of calculated and experimental data for each model in the speed $U$ at $(x / b=1,5,10,15,20,30,40,50)$

\section{Summary}

The calculation example is focus on low Reynolds number conditions, different turbulence models (SKE, AB, LB, LS, YS, AKN, CHC model) and two wall treatment for the near-wall shear flow forecasting and assessment.By comparing the simulation results with the experimental data to validate the turbulence model consistent with the degree of reliability with real physical flows.And 
by comparing the turbulence model of the calculated value of other physical quantities, to assess the advantages and disadvantages of each model.Table 1-3 summarizes the differences between the low Reynolds number turbulence two-equation model with the standard high Reynolds number turbulence two-equation model in FLUENT14.0, we can see the way of modification and the specific formula is various; as a result of the inspection scope widely enough, it is difficult to say that a better form.Nevertheless, it can be seen from the study, the role of molecular viscosity of the relative enhancement in low Reynolds number $k-\varepsilon$ turbulence.it be used to calculate the boundary layer problem, and compared to the standard $k-\varepsilon$ turbulence model in the wall is calculated accurately.But the low Reynolds number $k-\varepsilon$ turbulence models in the wall of the calculation requires more mesh nodes, so the computing time than using wall function about an order of magnitude.Therefore, in the case of calculating insufficient resources, standard turbulence model is applicable.

\section{Acknowledgment}

This work is supported by National Natural Science Foundation of China(11472095).

\section{Reference}

[1] Chang KC, Hsieh WD, Chen CS. A modified low-Reynolds-number turbulence model applicable to recirculating flow in pipe expansion. J Fluids Eng Trans ASME 1995;117:417-23.

[2] Launder BE, Sharma BI. Application of the energy-dissipation model of turbulence to the calculation of flow near a spinning disc. Lett Heat Mass Trans 1974;1:131-7.

[3] Abid R. Evaluation of two-equation turbulence models for predicting transitional flows. Int J Eng Sci 1993;31:831-40.

[4] Lam CKG, Bremhorst K. Modified form of the $k$-epsilon model for predicting wall turbulence. J Fluids Eng Trans ASME 1981;103:456-60.

[5] Yang Z, Shih TH. New time scale based $k-\varepsilon$ model for near-wall turbulence.AIAA J 1993;31:1191-8.

[6] Abe K, Kondoh T, Nagano Y. A new turbulence model for predicting fluid flow and heat transfer in separating and reattaching flows-I. Flow field calculations. Int J Heat Mass Transf 1995;37:139-51.

[7] S. Gorji , M. Seddighi, C. Ariyaratne, A.E. Vardy, T. O’Donoghue , D. Pokrajac, S. He .A comparative study of turbulence models in a transient channel flow.J Computers \& Fluids 2014;111-123.

[8] Sushil Kumar Rathore, Manab Kumar Das.Comparison of two low-Reynolds number turbulence models for fluid flow study of wall bounded jets.J International Journal of Heat and Mass Transfer 2013;365-380.

[9] Yuan Shi-Wei,Lai Huan-Xin.A Comparison of Five Low Reynolds Number $k-\varepsilon$ Models [J]Journal of Engineering Thermophysics,2014.(In Chinese)

[10] Arvind Pattamatta, Ghanshyam Singh.Assessment of Turbulence Models in the Prediction of Flow Field and Thermal Characteristics of Wall Jet [J] Frontiers in Heat and Mass Transfer 2012;

[11] Yohei Sato,Koichi Hishida,Masanobu Maeda.Effect of Dispersed Phase on Modification of Turbulent Flow in a Wall Jet.[J]Journal of Fuids Engineering1996;307-315. 